\title{
Viviane GRIVEAU-GENEST, Écriture du raffinement : l'esthétique des Lais de Marie de France
}

Sylvie Meyer

\section{(2) OpenEdition}

\section{Journals}

Édition électronique

URL : https://journals.openedition.org/ccm/8209

DOI : $10.4000 / \mathrm{ccm} .8209$

ISSN : 2119-1026

\section{Éditeur}

Centre d'études supérieures de civilisation médiévale/Université de Poitiers

\section{Édition imprimée}

Date de publication : 1 septembre 2021

Pagination : 296-297

ISBN : 978-2-490783-10-6

ISSN : 0007-9731

\section{Référence électronique}

Sylvie Meyer, "Viviane griveAu-genest, Écriture du raffinement : l'esthétique des Lais de Marie de France », Cahiers de civilisation médiévale [En ligne], 255 | 2021, mis en ligne le 01 septembre 2021, consulté le 05 février 2022. URL : http://journals.openedition.org/ccm/8209; DOI : https://doi.org/10.4000/ccm. 8209

\section{(c) (i) (9)}

La revue Cahiers de civilisation médiévale est mise à disposition selon les termes de la Licence Creative Commons Attribution - Pas d'Utilisation Commerciale - Pas de Modification 4.0 International. 
Viviane Griveau-Genest, Écriture du raffinement : l'esthétique des Lais de Marie de France, Mont-SaintAignan, Presses universitaires de Rouen et du Havre/Centre national d'enseignement à distance (Cours), 2018.

Ce petit livre est édité dans une série qui a pour objectif de présenter des synthèses " savantes et accessibles » pour la préparation des agrégations des lettres. Un bref survol du catalogue révèle que certains ouvrages ont été rédigés par des auteurs ayant auparavant publié des études sur le même thème, alors que d'autres sont dus à des universitaires travaillant habituellement dans des sphères quelque peu éloignées du sujet traité. Comme ces publications ne représentent pas l'aboutissement de travaux menés par des chercheurs dans leur domaine de connaissance, il est vraisemblable que les spécialistes y trouveront peu de matériau inédit.

Dans le cas présent, il s'agit de la rencontre entre un texte très lu et très commenté, les Lais de Marie de France, et une chercheuse expérimentée, quoique pas dans ce domaine spécifique. Viviane Griveau-Genest a été lauréate du prix de la société académique de Genève pour sa thèse de doctorat sur les sermons de Jean Gerson (Viviane GriveauGenest, L'esthétique du faire croire : étude littéraire des sermons français de Jean Gerson, thèse de doctorat en langue et littérature françaises, sous la direction de Marie-Christine GoMEz-GÉRAud et JeanYves Tilliette, Paris 10, 2017), et elle a déjà à son actif de nombreuses interventions et publications. Ses recherches portent principalement sur l'homélie, étudiée sous l'angle de l'esthétique, de la rhétorique ou de l'auctorialité, et en majorité sur des textes de la fin du Moyen Âge et de la Renaissance. L'a. n'avait pas publié à ce jour d'étude sur Marie de France ni sur d'autres textes du XII ${ }^{\mathrm{e}} \mathrm{s}$.

On aurait pu par conséquent s'attendre à une synthèse maîtrisée de la doxa en vigueur, sans réelle contribution personnelle, de ces ouvrages de commande qui, sous prétexte de faciliter l'approche des grands textes de la littérature, visent essentiellement à abréger le temps de travail de l'étudiant. Or il semble que l'a. a tenu à tracer son propre chemin à travers les Lais de Marie de France. Elle fait entendre une voix individuelle, nourrie à la critique contemporaine et portée par une écriture fluide et élégante.

Le livre est divisé en cinq parties thématiques. La première expose tout d'abord le contexte historique des Lais en mettant l'accent sur les mouvements intellectuels de la «Renaissance » du XII ${ }^{\mathrm{e}}$ s. Une longue dissertation est ensuite consacrée au lai comme genre littéraire, avant d'aborder la question de la structure du recueil et celle de l'auteur. En substance, l'a. reprend les conclusions d'études précédentes, en les discutant parfois, sa propre contribution consistant essentiellement à signaler des échos du contexte dans les Lais. On regrettera toutefois que le problème des manuscrits soit traité avec un peu de légèreté. Par exemple, elle récuse la fiabilité du ms. H 978 concernant l'organisation des textes dans le recueil. L'a. adhère à la thèse de $\mathrm{Ph}$. Ménard (1979), laquelle ne fait pas autorité en la matière, et occulte un pan conséquent de la critique textuelle à ce sujet. 
La deuxième partie est dédiée à « l'ombre des Celtes » dans les Lais. L'empreinte de la culture bretonne est étudiée à travers la langue, l'onomastique, la toponymie, les motifs et la mythologie. De nombreuses indications sont inventoriées et analysées dans une perspective interdisciplinaire. La discussion des sources reste cependant lacunaire. En se nourrissant presque exclusivement de l'ouvrage de Bernard Sergent, dont l'origine celtique des Lais est précisément la thèse principale, l'a. écarte les autres hypothèses sans réellement exposer de contre-arguments. La coloration celtique est manifeste, toutefois une analyse narratologique poussée aurait éclairé la question de l'hégémonie culturelle, indubitablement anglo-normande.

La troisième partie traite du merveilleux, ou plutôt de la forme particulière qu'il prend sous la plume de Marie de France. Alors que le sujet a longtemps occupé une place prépondérante dans les études médiévales, l'a. développe ici une argumentation originale qui implicitement le relègue au rang des ornements. Ayant constaté que, malgré la présence insistante du merveilleux, les lais ne suivent pas le schéma narratif des contes, et que de plus, le surnaturel n'est jamais au service d'une symbolique signifiante, elle propose d'y voir une forme épurée, stylisée par la littérature et rationalisée, voire christianisée dans certains lais.

La quatrième partie est entièrement consacrée au monde concret des Lais. L'a. relève toutes les occurrences susceptibles d'évoquer pour les lecteurs contemporains leur propre réalité : les relations vassaliques, le monde de la chasse et des tournois, le statut précaire des jeunes chevaliers et même des reflets de la politique. Les explications proposées sont remarquablement bien documentées. Les realia des Lais n'auraient pas seulement pour fonction de créer une connivence avec les lecteurs et de renforcer la vraisemblance, mais joueraient un rôle structurant au niveau de l'intrigue - rôle qu'il resterait d'ailleurs à expliciter.

Enfin, la cinquième partie se penche sur le « jeu de l'amour ». C'est certainement un des aspects des Lais qui a été le plus étudié et l'a. parvient à intégrer les principales thèses à ses propres analyses. Le système des objets, en particulier, est étudié avec beaucoup de finesse. La peinture des sentiments, du désir, de l'érotisme et des figures féminines sont mis en exergue par petites touches, en naviguant d'un lai à l'autre. L'a. ne propose pas en conclusion de vision unifiée du paradigme amoureux, préférant considérer l'ensemble des récits comme différentes variations autour des relations femmes-hommes, cependant « reconfigurées de manière plus égalitaires » (p. 121).
La conclusion constitue le véritable point d'orgue de l'ouvrage. Exploitant la métaphore filée du contrepoint musical, l'a. livre sa propre vision des Lais. En fin de parcours, les ambiguités et les contradictions des lais sont savamment dépassées par le biais de l'élaboration d'une « esthétique faite de discontinuité, de ruptures et de reprises » (p. 129). Il est aussi beaucoup question du plaisir partagé de l'écriture et de la lecture, délectation qui transparaît dans l'écriture même de l'a.

La partition thématique des chapitres impose une lecture quelque peu superficielle et décousue des lais : on saute de texte en texte, au gré des analogies, sans jamais aborder un lai dans sa perspective narrative intégrale. Cette approche est justifiée dans la mesure où l'a. considère les Lais comme une « œuvre ouverte » (p. 39). Partant, elle se réclame de la liberté critique qui « augmente le sens », piochant dans le texte et le métatexte les éléments susceptibles d'alimenter sa lecture. Les extrapolations sont fertiles, créatives, dynamiques, si tant est qu'on garde à l'esprit que le passage à l'extradiscursif en apprend davantage sur le locuteur que sur l'objet de son discours. Cela étant, le parti pris de la subjectivité est tout à fait légitime. Là où le bât blesse, en revanche, c'est qu'on ne parvient pas à se libérer du sentiment diffus que l'a. n'est pas parvenue à une connaissance intime des Lais, et qu'elle choisit un peu trop souvent de promouvoir l'ambivalence et la contradiction au lieu d'en questionner les motifs. Par exemple, après avoir insisté sur le programme d'écriture du Prologue (p. 9), elle n'hésite pas à parler de " désinvolture » (p. 124) dans la réalisation du projet. C'est peut-être de ce même goût assumé pour l'incompatibilité que jaillissent des formules comme une « logique de mimesis floue » (p. 10), ou un " autre régime de spatialité [...] qui s'affranchit aisément des lourdeurs de l'espace-temps » (p. 56). On trouve aussi quelques méprises, comme d'affirmer au sujet des titres que nihtegale est " écrit de façon plutôt phonétique 》 (p. 49), alors que dans un même temps, l'a. omet de mentionner l'incongruité de gotelef, autrement singulier.

Ces quelques réserves mises à part, l'ouvrage reste une lecture stimulante, toujours intelligente, et il aurait mérité d'être servi par un meilleur toilettage du texte.

Sylvie MeYer

EA 4451 - Centre de recherche bretonne et celtique / Université de Bretagne Occidentale 